\title{
On the kinetics of solvate formation through mechanochemistry
}

DOI:

10.1039/C8CE00871J

Document Version

Accepted author manuscript

Link to publication record in Manchester Research Explorer

\section{Citation for published version (APA):}

Hasa, D., Pastore, M., Arhangelskis, M., Gabriele, B., Cruz-cabeza, A. J., Rauber, G. S., Bond, A. D., \& Jones, W. (2018). On the kinetics of solvate formation through mechanochemistry. CrystEngComm.

https://doi.org/10.1039/C8CE00871J

\section{Published in:}

CrystEngComm

\section{Citing this paper}

Please note that where the full-text provided on Manchester Research Explorer is the Author Accepted Manuscript or Proof version this may differ from the final Published version. If citing, it is advised that you check and use the publisher's definitive version.

\section{General rights}

Copyright and moral rights for the publications made accessible in the Research Explorer are retained by the authors and/or other copyright owners and it is a condition of accessing publications that users recognise and abide by the legal requirements associated with these rights.

\section{Takedown policy}

If you believe that this document breaches copyright please refer to the University of Manchester's Takedown Procedures [http://man.ac.uk/04Y6Bo] or contact uml.scholarlycommunications@manchester.ac.uk providing relevant details, so we can investigate your claim.

\section{OPEN ACCESS}




\section{Journal Name}

\section{ARTICLE}

\section{On the Kinetics of Solvate Formation through Mechanochemistry}

Received 00th January 20xx, Accepted 00th January 20xx

DOI: $10.1039 / x 0 x \times 00000 x$

www.rsc.org/

\author{
Dritan Hasa, ${ }^{a}+$ Mariana Pastore, ${ }^{b}$ Mihails Arhangelskis, ${ }^{c}$ Benjamin Gabriele, ${ }^{d}$ Aurora J. Cruz- \\ Cabeza, ${ }^{d}+$ Gabriela Schneider Rauber, ${ }^{e}$ Andrew D. Bond, ${ }^{e}$ and William Jones ${ }^{e}$
}

Theophylline:2-pyrrolidone mono (1:1) and sesqui (2:3) solvates have been discovered through variable-amount liquidassisted grinding (VALAG). The structures and stability of the solvates and the kinetics involved in their formation are investigated both experimentally and theoretically. Ex-situ studies reveal a delayed appearance of the sesquisolvate, and show that sesquisolvate formation occurs via the monosolvate rather than directly from pure theophylline. Theoretical calculations show that the obtained solvates are the thermodynamic products corresponding to the reactant ratio. The kinetics of the transformations were found to be related to the energy required to cleavage the crystals through the softest planes. This was quantified by means of attachment energy calculations.

\section{Introduction}

In many stages of chemical and pharmaceutical industrial processing, substances are exposed to a series of solution-based procedures such as crystallisation, wet granulation, lyophilisation, spray-drying etc. Contact between a solid and the solvent(s) is unavoidable under such conditions, and as a consequence, solvent molecules frequently become "entrapped" within the crystal structure of processed substances to form solvates. ${ }^{1}$ New solvates are discovered either by chance during a specific manufacturing process $^{2}$ or through systematic polymorph screening programs. ${ }^{3,4}$ Most screening methods are based on solution crystallisation techniques (i.e. in the presence of solvent excess) where only the most stable solvate is likely to be obtained. Other solvates with different stoichiometries are usually found in subsequent investigations, for example during desolvation studies. Such methods seldom provide information about the mechanisms of solvate formation. Alternative techniques, such as high-pressure crystallisation $^{5}$ and freeze-drying ${ }^{6}$ have also been proposed for discovering new solvates.

In this study, we explore the use of mechanochemistry, in particular liquid-assisted grinding (LAG), as a systematic screening method for

a. Leicester School of Pharmacy, De Montfort University, the Gateway, LE1 9BH Leicester, United Kingdom.

${ }^{b .}$ Department of Chemical and Pharmaceutical Sciences, University of Trieste, P.le Europa 1, 34127 Trieste, Italy.

Department of Chemistry, McGill University, 801 Sherbroke Street West,

Montreal, Quebec H3A OB8, Canada

${ }^{d .}$ School of Chemical Engineering and Analytical Science, University of Manchester,

Oxford Road, M13 9PL, Manchester, United Kingdom.

e. Department of Chemistry, University of Cambridge, Lensfield Road, CB2 1EW Cambridge, United Kingdom

$+\quad$ Corresponding authors: dritan.hasa@dmu.ac.uk aurora.cruzcabeza@manchester.ac.uk

Electronic Supplementary Information (ESI) available: [details of any supplementary information available should be included here]. See DOI: $10.1039 / \mathrm{x} 0 \times x \times 0000 \mathrm{x}$ the production of solvates of varying stoichiometry for a given substance-solvent system. We also explore possible formation mechanisms and their kinetics. Our approach is based on the well documented success of mechanochemistry as a screening tool for novel multicomponent crystals (including co-crystals) that show stoichiometric variations and polymorph diversity. ${ }^{7-10}$ The significant success of this technique for discovering different solid forms (including new crystal forms that cannot be obtained easily by alternative approaches) can, in part, be attributed to the large number of variables influencing the product outcome. ${ }^{11}$ For instance, during LAG synthesis high degrees of supersaturation ${ }^{12,13}$ are always present since only small amounts of liquid are used. This would therefore increase the role of other process-related variables able to cover a wide range of crystallization space, often enabling the formation of less stable forms. ${ }^{11}$

Fig. 1. Chemical structures of THEO (left) and PYR (right).

In the case of the discovery of solvates, mechanochemistry has<smiles>Cn1c(=O)c2[nH]cnc2n(C)c1=O</smiles><smiles>O=C1CCCN1</smiles>

rarely been used due to the common belief that solvates may not be stable under mechanochemical stress. Previous work in this area, however, clearly demonstrated the possibility of obtaining solvated forms through grinding, or by combining mechanochemistry and solution synthesis ${ }^{14}$, for both single ${ }^{15-17}$ and multicomponent solids (solvated co-crystals). ${ }^{18,19}$ To the best of our knowledge, however, only two systematic studies with regard to the synthesis of solvates have been reported so far. Specifically, 
LAG was used to obtain different stoichiometric hydrates of the same inorganic molecule, ${ }^{20}$ and to control hydration/dehydration of a metal organic framework. ${ }^{16}$ In both cases, the applied approach relied on the modification of water activity by the addition of another solvent such as ethanol or DMF.

Here, we report a systematic one-solvent mechanochemical screening for different stoichiometric solvates of theophylline (THEO) with 2-pyrrolidone (PYR, Fig. 1) by means of variableamount LAG (VALAG). The rationale behind this strategy is based on a typical constant-temperature stability phase diagram for solvates; ${ }^{21}$ a specific solvate must be stable over a range of solvent activities, while outside this range another solid (a higher/lower solvate or the non-solvated material) will be the preferred form. ${ }^{22}$ Indeed, we believe that the activity of the solvent can be modified during mechanochemical solvate formation by changing the stoichiometric ratio of reactants and without the addition of another solvent. This approach is similar to the preparation of stoichiometric co-crystals: different co-crystal forms can be obtained in a mechanochemical synthesis by changing the stoichiometric ratio of the starting materials. ${ }^{23}$ Beyond demonstrating the usefulness of VALAG in the discovery of stoichiometric solvates, we also use computational methods to investigate the kinetics of solvation formation on grinding, and propose that the reaction are correlated with the attachment energies of the softest crystal planes.

\section{Experimental}

\section{Materials}

Anhydrous THEO Form II, PYR and the other solvents used during preliminary screening were purchased from SigmaAldrich Company, Ltd. (Gillingham, UK), and used without further purification. THEO Form IV, the most stable polymorph of THEO, was obtained via a solvent-mediated phase transformation $^{24}$ from THEO Form II using the method described by Bobrovs et al. ${ }^{25}$ Four grams of THEO Form II were stirred in $100 \mathrm{~mL}$ of methanol (Acros Organics, Extra Dry, over Molecular Sieves) for 50 days at a constant temperature of 23 $\pm 0.2 \circ$ C. Every few days, solid samples were retrieved and analysed via PXRD measurements. Form IV started to appear after 30 days and full conversion was achieved after 40 days (ESI).

\section{Grinding experiments}

THEO solvates were prepared mechanochemically by grinding 1.11 $\times 10^{-3}$ mole (approximately $200 \mathrm{mg}$ of solid) of anhydrous THEO in the presence of different stoichiometric ratios of PYR, ranging from 1:0.1 (corresponding to $8 \mu \mathrm{l}$ of PYRR) to 1:4 (corresponding to 332 $\mu$ l of PYR). Each experiment was repeated twice. The weighed materials were added to a $15 \mathrm{ml}$ stainless steel jar containing two (stainless steel) grinding balls of $7 \mathrm{~mm}$ diameter each. Although PYR is not a highly volatile solvent, snap closed grinding jars were used to minimize liquid evaporation. The mixture was subsequently ground for varying times in a Retsch MM200 grinding mill at a frequency of $25 \mathrm{~Hz}$. In the case of the interconversion studies, approximately $200 \mathrm{mg}$ of pre-formed THEO monosolvate or sesquisolvate were neat ground for up to $180 \mathrm{~min}$ at $25 \mathrm{~Hz}$. The process was paused for $5 \mathrm{~min}$ every $60 \mathrm{~min}$ of use to avoid significant temperature increase. In the cases where mixtures were used, the total amount of the solid added to the jar was $200 \mathrm{mg}$.

\section{Powder X-ray diffraction (PXRD) studies}

PXRD analyses were performed at room temperature using a Panalytical X'Pert Pro Diffractometer with Ni-filtered Cu-K $\alpha$ radiation (wavelength $1.5418 \AA$ ) equipped with an RTMS X'celerator detector. For each measurement, about 20 to $30 \mathrm{mg}$ of powder was gently pressed on a glass slide to give a flat surface and subsequently analysed. The data were collected in the 2 theta range $3-40^{\circ}$ using a step size of $0.0334^{\circ}$ and a scan speed of $0.142 \%$ s. For each analysis the total number of steps was 1107 and the total time was $5 \mathrm{~min}$.

\section{Quantitative PXRD studies}

PXRD patterns were used to determine the relative amounts of each component in mixtures of THEO and the PYR solvates. Quantitative phase analysis was performed using TOPAS Academic 4.1 software. First, the powder pattern of NIST standard reference material $660 \mathrm{~b}$ (Lanthanum hexaboride) was collected to obtain the instrumental contribution to the diffraction peak shape. The peak shape was modelled with a pseudo-Voigt peak-shape function with a correction for axial divergence. The room-temperature unit-cell parameters of THEO, THEO:PYR monosolvate and THEO:PYR sesquisolvate were refined against the powder patterns of the pure materials. The peak-shape parameters obtained from the reference standard, as well as the unit-cell parameters of the THEO phases were then kept fixed during the quantification procedure. The quantitative composition of the mixtures was determined by refining the scale factors corresponding to each crystalline phase present in the mixture. The effects of crystallite size broadening were modelled with Lorentzian broadening terms. The background was modelled with a 6-term Chebyshev polynomial.

\section{Single crystal structure analysis}

Single-crystal X-ray diffraction data were collected for THEO:PYR monosolvate and THEO:PYR sesquisolvate at 180 (2)K using a Bruker D8-QUEST PHOTON-100 instrument, equipped with an Incoatec I $\mu \mathrm{S} \mathrm{Cu}$ microsource. Analysis of the monosolvate was straightforward. For the sesquisolvate, crystals were found to be twinned. Two domains were identified in the diffraction pattern, related by a 2 -fold rotation around the $a$-axis of the unit cell. Integration and scaling was carried out using both components, then the structure was refined against data from the stronger of the two components, plus overlapping data. The refined batch scale factor suggested a $c a 10 \%$ contribution of the second twin component to the overlapped reflections. Details of the crystallographic information are provided in the ESI.

\section{Lattice energy calculations}

The crystal structures of THEO Form II (BAPLOT06) ${ }^{26}$ and THEO Form IV (BAPLOT03) ${ }^{27}$ were retrieved from the Cambridge Structural Database. The crystal structures of the THEO:PYR solvates were taken from our XRD analysis. All crystal structures were geometry optimised using periodic DFT-d as implemented in VASP version 5.4.1. ${ }^{27-28}$ The PBE functional ${ }^{30}$ was used together with PAW 
pseudopotentials $^{31,32}$ and the dispersion corrections of Grimme (second version, PBE-d). ${ }^{33}$ Unit-cell volumes were optimised together with atomic positions. An energy cut-off of $520 \mathrm{eV}$ was applied for the plane-waves and structural relaxations were stopped when the calculated force on every atom was less than $0.005 \mathrm{eV} / \mathrm{A}$. The Brillouin zone was sampled using the MonkhorstPack approximation on a grid of k-points separated by approximately $0.04 \AA^{-1}$. The resulting unit-cell electronic energies of the relaxed crystal structures were normalised by the number of molecules in the simulation cell. In the case of the solvates, normalisation was done per 1:1 THEO-PYR pairs for the monosolvate and for 1:1.5 THEO-PYR pairs for the sesquisolvate. This resulted in values of crystal electronic energies normalised per molecule of theophylline ( $\left.E_{e-c r y s}\right)$.

In a separate simulation, a single molecule of THEO was placed in a large box $(25 \times 25 \times 25 \AA)$. The geometry of THEO was then relaxed keeping the volume of the simulation cell fixed, using the same computational model. The same simulation was also performed for PYR. These calculations provide the electronic energies in the gasphase $\left(E_{e-g a s}\right)$ for THEO and PYR. Lattice energies were calculated as the difference between the normalised electronic energies of the components in the crystals $\left(\mathrm{E}_{\mathrm{e}-\mathrm{crys}}\right)$ minus the electronic energy of the components in the gas-phase $\left(\mathrm{E}_{\mathrm{e} \text {-gas }}\right)$. The equations below were used for the different crystal systems.

$E_{\text {latt }}(T H E O$ form II $)=E_{\text {e-crys }}\left(\right.$ THEO form II) $-E_{\text {e-gas }}($ THEO $)$

$E_{\text {latt }}($ Monosolvate $)=E_{\text {e-crys }}($ Monosolvate $)-E_{\text {e-gas }}(T H E O)-E_{\text {e-gas }}(P Y R)$

$E_{\text {latt }}($ Sesqui-solvate $)=E_{\text {e-crys }}($ Sesqui-solvate $)-E_{\text {e-gas }}($ THEO $)-1.5 E_{\text {e-gas }}(T H E O)$

\section{Energy frameworks}

Crystal Explorer version $17.5^{34}$ was used for computing moleculemolecule interactions in the crystal structures of pure THEO and the THEO:PYR monosolvate. The CE-B3LYP model for molecular pair energies was used as derived from $B 3 L Y P / 6-31 G(d, p)$ electron densities. ${ }^{35,36}$ Energy frameworks ${ }^{37}$ were then visualised within Crystal Explorer using an energy cut-off of $12 \mathrm{~kJ} / \mathrm{mol}$.

\section{Attachment energy calculations}

Crystal Explorer energy Frameworks were used to identify the crystallographic cleavage planes for the different structures. Cleavage planes are those through which crystals are easier to break; they have small attachment energies. We identified the following cleavage planes for the different structures: i) (200) for THEO form II, ii) (002) for THEO form IV, iii) (001) for the THEO:PYR monosolvate and iv) (020) for the sesquisolvate. Slices of these crystallographic faces were generated manually from the crystal structures. For this, two of the cell parameters were kept constant whilst the third one (that being the direction perpendicular to the plane under study) was imposed to be $40 \AA$. Excess molecules not belonging to the single slice under evaluation were deleted. The energy of a single slice of molecules $\left(E_{\text {slice }}\right)$ was then computed using PBE-d. If several slices were possible due to different choices of origins, the energetics of all of them were computed and the stability of the most stable slice is reported. Attachment energy for the particular face was calculated as the difference between the lattice energy minus the slice energy $\left(E_{a t t}[h k l]=E_{\text {latt }}-E_{\text {slice }}[h k l]\right)$.

\section{Results and Discussion}

\section{Preliminary grinding experiments}

The experimental dataset was obtained using THEO $^{\ddagger}$ as a model compound and PYR as a solvent ${ }^{\S}$ (Fig. 1). During a preliminary screening, THEO Form II and PYR were ground for $60 \mathrm{~min}$ in 4 different molar ratios, namely 1:0.5, 1:1, 1:1.5 and $1: 2$. Ground products were analysed by powder X-ray diffraction (PXRD), differential scanning calorimetry (DSC) and thermogravimetric analysis (TGA, see ESI for full details). Diffraction data suggests that the product formed by grinding THEO Form II with PYR in a 1:0.5 molar ratio contains the original Form II THEO together with a new solid phase (Fig. 2). This new phase is identical to that obtained on grinding THEO Form II and PYR in 1:1 ratio. PXRD patterns of the products containing THEO Form II and PYR in $1: 1.5$ and $1: 2$ ratios are also different (Fig. 2). TGA analyses of the products (reported in the ESI) revealed weight loss in all cases, suggesting that the new solid forms obtained are solvates of THEO and PYR.

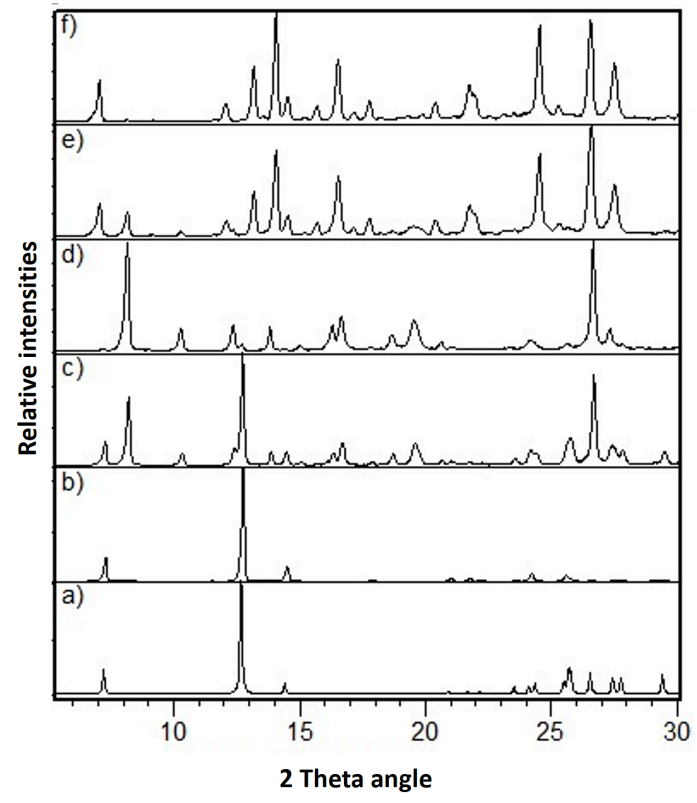

Fig. 2. PXRD pattern of a) calculated THEO Form $\|^{26}$, b) experimental THEO Form $\mathrm{Il}, \mathrm{c}$ ), d), e) and solid products obtained by grinding THEO and PYR in 1:0.5, 1:1, 1:1.5 and 1:2 molar ratios, respectively.

\section{Crystallisation experiments and new solvate structures}

Following the VALAG experiments, we performed a series of cooling crystallisation experiments where supersaturated solutions of THEO Form II in PYR were seeded with the mechanochemical products. These seeding crystallisations resulted in single crystals that were confirmed by single-crystal XRD (ESI) to be THEO:PYR monosolvate (1:1) and a THEO:PYR sesquisolvate (1:1.5). 
a)

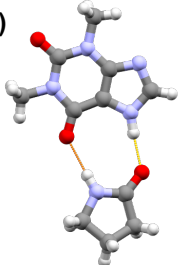

Fig. 3. Visualisation of the asymmetric unit of the THEO:PYR monosolvate (a) and sesquisolvate (b). Dashed lines illustrate hydrogen bonds.

The monosolvate consists of one molecule of THEO and one molecule of PYR in the asymmetric unit. These molecules form discrete $R_{2}{ }^{2}(9)$ heterodimer units, where the molecules interact via $\mathrm{N}-\mathrm{H}^{\cdots} \mathrm{O} \mathrm{O}$ hydrogen bonds (Fig. 3a). The asymmetric unit of the sesquisolvate structure consists of two molecules of THEO and three molecules of PYR. Two of the PYR molecules form a $R_{2}^{2}(8)$ homodimer, which further interact with two THEO molecules via $\mathrm{N}-\mathrm{H}^{\prime} \cdot \mathrm{O}$ hydrogen bonds. The third PYR molecule forms a discrete centrosymmetric $\mathrm{R}_{2}{ }^{2}(8)$ homodimer without further $\mathrm{H}$-bonding interactions (Fig. $3 \mathrm{~b}$ ). Thus, the two solvates might be viewed in structural terms to comprise THEO together with isolated molecules of PYR (in a 1:1 ratio), or THEO together with PYR dimers (in a 4:3 ratio).

\section{VALAG experiments: Thermodynamic studies}

Following the initial grinding experiments, a more extensive VALAG screening was performed where the stoichiometric ratio between THEO Form II and PYR was incrementally increased from 1:0.1 to 1:4. The results suggested that three forms were obtained, namely non solvated Form II THEO, THEO:PYR monosolvate and THEO-PYR sesquisolvate. The quantitative analysis of the PXRD data for the performed experiments is presented in Fig. 4. Clearly, pure THEO and its monosolvate coexist when the stoichiometry between reagents is lower than 1:1, while THEO:PYR sesquisolvate appears only at stoichiometries of 1:1.1 and above, becoming the predominant solid phase when the molar ratio of starting materials reaches 1:1.5 or higher (Fig. 4). Pure sesquisolvate can only be obtained under the applied conditions when THEO and PYR are ground in 1:1.7 (or higher) molar ratios.

We note that all the VALAG experiments presented in Fig. 4 were performed under prolonged milling times. The materials were ground for $60 \mathrm{~min}$, after which, the PXRD data were measured. We also note that none of the experiments produced a mixture where THEO and the sesquisolvate coexisted. In order to explore the thermodynamic stability of these systems further, a series of additional grinding experiments were performed. The pure THEO:PYR monosolvate and sesquisolvate were neat-ground for 3 hours and no changes in solid form were observed (ESI). Additionally, no conversion was observed when THEO:PYR monosolvate was co-ground with pure Form II THEO or with THEO:PYR sesquisolvate. When grinding THEO:PYR sesquisolvate with pure Form II THEO, however, the THEO-PYR monosolvate was obtained (see ESI for further details).

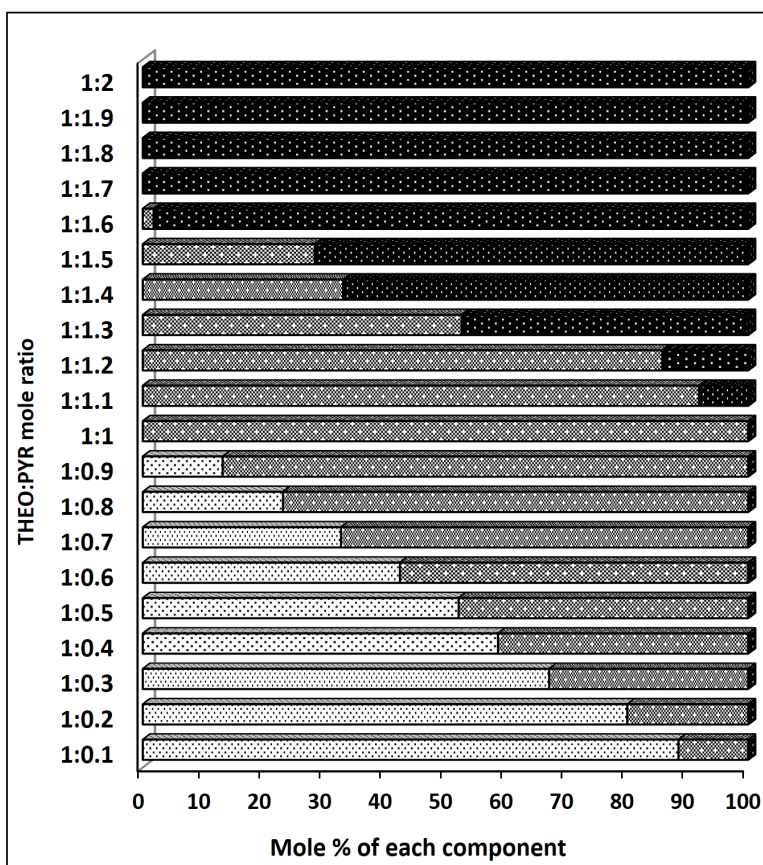

Non-solvated Theo Monosolvate S Sesquisolvate

Fig. 4. Quantitative PXRD analysis of products prepared mechanochemically by using different molar ratio between THEO:PYR.

\section{Energetics of solvate formation}

In an attempt to understand the energetics driving solvate formation and stoichiometry changes for these systems, we performed a series of lattice calculations with a PBE-d model. If we view the solvation process as a supramolecular reaction, we can calculate the reaction energy or energy gain $\left(E_{\text {gain }}\right)$ as the difference between the energy of the products minus the energy of the reactants weighted by their stoichiometries (Equation 1). This approach has been used in the past to explain solvate ${ }^{38}$ and hydrate $^{39}$ formation, as well as stoichiometric observations. ${ }^{40,41}$ We calculated the energy gain for the different reactions involved and various stoichiometric ratios using computed lattice energies of products and reactants. Since PYR is a liquid at room temperature, using the lattice energy here is an approximation, so instead we used the experimental heat of vaporisation. ${ }^{40}$

$$
E_{\text {gain }}=\sum x_{\text {product }} E_{\text {product }}-\sum x_{\text {reactant }} E_{\text {reactant }}
$$

The energy gains for all possible outcomes of a supramolecular reaction between THEO Form II and PYR and for a series of THEO:PYR stoichiometries are reported in Fig. 5. Possible reaction products are: a) only the 1:1.5 solvate forms -dotted red lines-, b) only the 1:1 solvate forms -black line-, c) the 1:1 solvate forms up to 1:1 stoichiometries and then formation of 1:1.5 solvate -green line-, and d) no solvate formation - purple line. 


\section{PYR:THEO Stoichiometric Ratio}

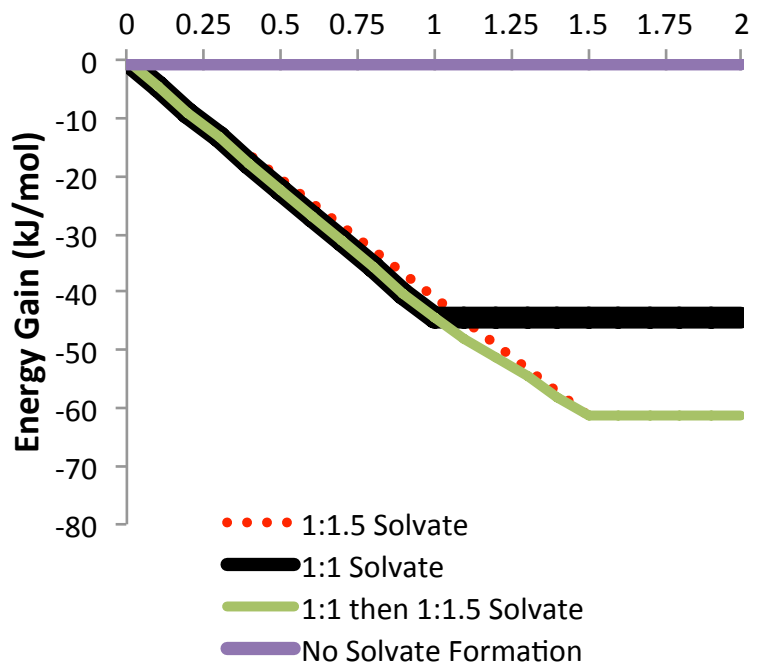

Fig. 5. Plot of energy gain as a function of PYR:THEO stoichiometric ratio for all possible reaction products.

As seen in Fig. 5, there is a favourable (negative) energy gain in forming the THEO:PYR solvates. This calculated energy gain is also supported by the DSC analysis of the experimental solvates (ESI). The model also predicts that in the 0:1-1:1 THEO:PYR stoichiometric range, the thermodynamically stable product of the reaction is the 1:1 solvate (black or green lines). Above the 1:1 stoichiometry, a mixture of the mono and the sesquisolvate is the most stable product. The calculations, therefore, support that the mixtures obtained under prolonged milling times using VALAG always correspond to the thermodynamically stable product. ${ }^{42}$ In the current system, the thermodynamic product outcome is a function of the chemical composition, so below 1:1 THEO-PYR ratios the 1:1 solvate alone is favoured whilst above the $1: 1$ ratios a mixture of the mono and sesquisolvate is the thermodynamically stable product.

VALAG experiments: Kinetics studies
In an attempt to explore the kinetics of the solvate formation, VALAG products of THEO Form II ground with various amounts of PYR were analysed as a function of time. Quantitative phase analysis of PXRD patterns (Fig. 6) revealed that Form II THEO always transforms first into the monosolvate, independent of the molar ratio of the reactants. Such transformation is almost instantaneous in all cases $190 \%$ of Form II THEO transforms into THEO:PYR monosolvate within 30 seconds of grinding, Fig. 6). The sesquisolvate, however, requires substantial mechanical activation to form. In stoichiometries 1:1.5 and above, THEO Form II fully converts to the monosolvate first and then requires substantial further grinding time to convert to the sesquisolvate. This indicates that, despite the sesquisolvate being the thermodynamically stable form at chemical compositions of $1: 1.5$ and above, the sesquisolvate is harder to access and is produced via the monosolvate as an intermediate state. It is also worth noting that excess liquid (PYR) seems to accelerate the kinetics of sesquisolvate formation. For example, after 10 minutes of grinding a sesquisolvate yield of $35 \%$ is achieved for the 1:1.5 stoichiometry whilst $50 \%$ yield is achieved for the 1:1.7 stoichiometry. Full conversion to the sesquisolvate is achieved in 30 mins for 1:1.7 stoichiometry whereas it requires almost 120 mins for the $1: 1.5$ stoichiometry.

\section{Energy Frameworks and Soft Planes}

We proceeded to calculate molecule-molecule interactions within the two reactant forms (THEO Form II as well as the THEO:PYR monosolvate) using Crystal Explorer. The strongest interaction in pure THEO Form II is the $\mathrm{H}$-bonded chain (-92 $\mathrm{kJ} / \mathrm{mol}$ per molecule in a $\mathrm{H}$-bonded chain) followed by the stacks $(-68 \mathrm{~kJ} / \mathrm{mol}$ per molecule in an infinite stack). In the monosolvate, however, THEO:THEO stacks are the most stabilising interaction $(-94 \mathrm{~kJ} / \mathrm{mol}$ per molecule inserted in an infinite stack) followed by the THEO:PYR $\mathrm{H}$-bonded dimer $(-80$ $\mathrm{kJ} / \mathrm{mol})$. In an attempt to rationalise the fast kinetics of the monosolvate formation versus the very slow kinetics of the sesquisolvate formation, we proceeded to identify the softest crystallographic planes in Form II THEO and the monosolvate. This was done with the help of crystal energy frameworks (Fig. 7).
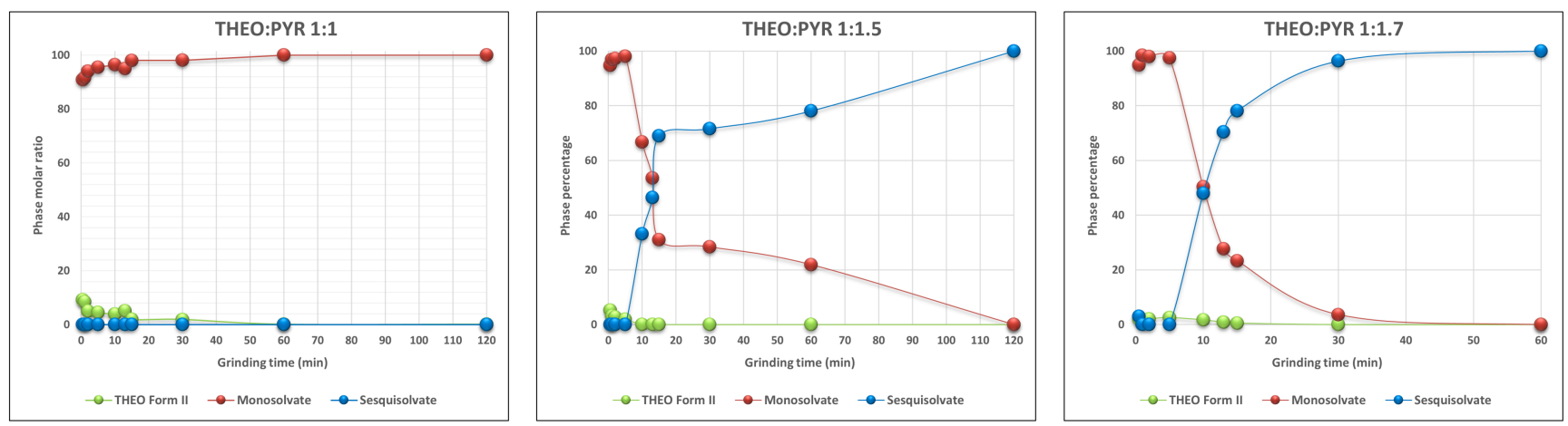

Fig. 6. Ex-situ experiments performed using different stoichiometric ratios between THEO and PYR. 

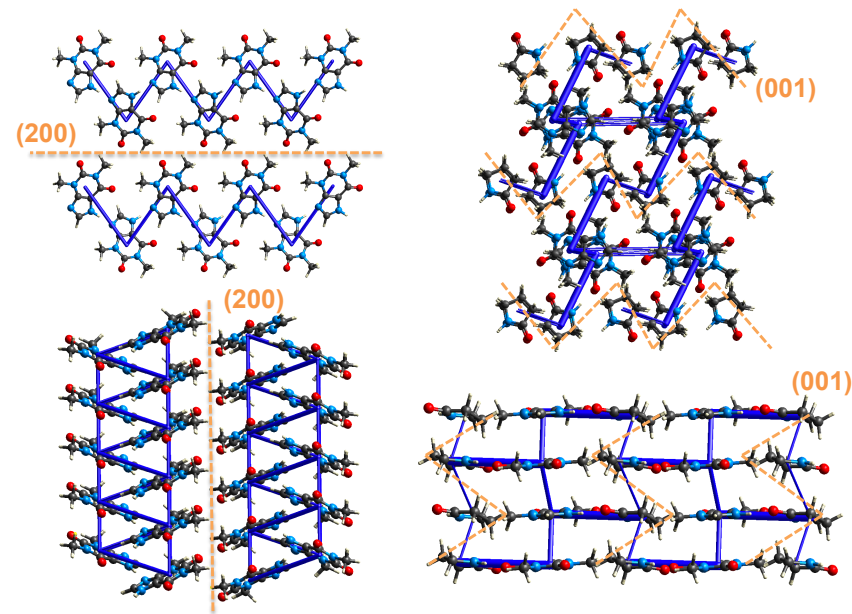

Fig. 7. Energy frameworks for THEO Form II (left) and the THEO:PYR monosolvate (right). The softest crystallographic directions are delimited by the orange dashed lines.

Crystals are more likely to fracture across slip planes on grinding. This would result in new surfaces being exposed and available to be in physical contact with the solvent for solvation to occur. Softest planes are those containing the weakest molecule-molecule interactions and correspond to the slip planes in the crystal structure. Pure THEO forms layered structures with strong $\mathrm{H}$-bonds and strong stack interactions (Fig. 7, left). The layer-to-layer packing is sustained by weaker van der Waals interactions (Fig. 7, (200) plane) and is the softest crystallographic direction. It would be expected, therefore, that this surface will be more easily exposed on grinding. The monosolvate, however, has a more complex 3D energy framework than THEO Form II (Fig. 7, right). In this structure, the THEO:PYR H-bonded dimer interacts strongly (Fig. 7, right) and also forms strong THEO:THEO stacks. These stacks interlock with each other through the stacking of PYR molecules. The stacking of PYR molecules is not as strong as the stacking of THEO, their interaction energy being $-22.3 \mathrm{~kJ} / \mathrm{mol}$ per stack $(-44.6 \mathrm{~kJ} / \mathrm{mol}$ per molecule of PYR inserted in an infinite stack). The (001) plane constitutes the softest crystallographic direction in the monosolvate (Fig. 7, right). We note that the softest plane of the monosolvate is much more strongly bound than the softest plane of THEO.

\section{Attachment energy calculations}

To further explore the kinetics of solvate formation, we computed the attachment energies of the softest crystallographic directions in pure THEO and the THEO:PYR monosolvate. The computed attachment energies for the (200) plane of THEO and the (001) plane for the THEO:PYR monosolvate are given in Table 1 . From the energy gain calculations, we know that solvation is driven by thermodynamics. We now postulate that the activation energy required to form the solvates, at least for this particular system, may be related to the ease of exposure of key surfaces of the reactants. The attachment energy of the (200) THEO is very small, $-19.8 \mathrm{~kJ} / \mathrm{mol}$. Upon grinding THEO and PYR in the
$0: 1-1: 1$ range of stoichiometries, formation of the monosolvate forms is driven by thermodynamics, and kinetics are very fast due to the small activation energy required. In THEO:PYR ranges of stoichiometries from 1:1.5 to 1:2, the sesquisolvate also forms, driven by thermodynamics. However, the activation barrier for this solvation must be much higher since achieving full conversion to the sesquisolvate requires significantly more grinding time, and passes through the monosolvate as an intermediate state. This is consistent with our hypothesis since the attachment energy of the softest surface in the monosolvate is much lower, $-72.5 \mathrm{~kJ} / \mathrm{mol}$. More energy is required to expose the surface, the activation energy of the solvation will be higher and thus result in much slower kinetics.

Table 1. Summary of attachment energies for the softest cleavage planes in THEO Form II and the THEO:PYR monosolvate.

\begin{tabular}{|c|c|}
\hline & $\begin{array}{c}\text { Attachment Energy } \\
\text { per mol of THEO (kJ/mol) }\end{array}$ \\
\hline THEO Form II, (200) & -19.8 \\
\hline Monosolvate, (001) & -72.5 \\
\hline
\end{tabular}

\section{Further kinetic studies: Solvation of Form IV vs. Form II}

In an attempt to expand upon the apparent link between the kinetics of solvate formation upon grinding and the attachment energies of softest faces in the reactants, we proceeded to investigate the kinetics of THEO:PYR monosolvate formation when the reactant THEO is in polymorphic Form IV rather than Form II. Prior to any experiment, we computed the lattice energy and the relevant attachment energies for THEO Form IV. The results suggested that the total lattice energy of Form IV differed from Form II by only $0.6 \mathrm{~kJ} / \mathrm{mol}$. The strong thermodynamic drive for solvate formation with PYR is therefore approximately equivalent for both forms. The attachment energy for the (002) face of Form IV THEO, the softest cleavage plane, was computed to be -35 $\mathrm{kJ} / \mathrm{mol}$. The softest cleavage plane in Form IV is thus more strongly bound than that of Form II, but still significantly softer than the monosolvate (001) face (Table 1). Thus, this calculation suggests that crystals of Form IV should convert to the monosolvate more slowly than crystals of Form II.

In an attempt to verify this prediction experimentally, we ground for 1 minute using the ball mill THEO(Form II):PYR and THEO(Form IV):PYR in 1:1 molar ratio. A visual inspection of the resulting PXRD patterns suggested that high amounts of monosolvate were produced in both cases under the experimental conditions mentioned above, while only traces of original THEO could be detected. In order to slow the kinetics to be able to differentiate the reactivity of the two polymorphic forms, we performed a series of additional experiments where reactants were mixed manually with the aid of a spatula for 1 minute. Upon such small mechanical activation, Form II transformed to the monosolvate almost completely whilst only a partial conversion was observed in the case of Form IV (ESI). Finally, the exposure of THEO Form II 
and Form IV to PYR vapour for 60 mins, promoted a full conversion of Form II to the monosolvate whilst no conversion was observed for THEO Form IV (ESI). This suggests that PYR is able to penetrate the crystal of THEO form II most likely through the (200) cleavage planes from the available morphological surfaces. These experiments appear to corroborate the suggestion that the nature and attachment energies of relevant cleavage planes in the reactants are key for the kinetics of mechanochemical solvation reactions.

\section{Conclusions}

We have reported the formation of two stoichiometric THEOPYR solvates, first obtained mechanochemically and subsequently by cooling crystallisation in the presence of seeds. The study demonstrates the value of VALAG as an efficient screening technique for the production of novel stoichiometric solvates. Such a methodology, therefore, can be used for organic materials as well as inorganics. Products obtained mechanochemically may then be used to seed supersaturated solutions for crystal growth.

Theoretical calculations have shown that the formation of these THEO:PYR solvates is driven by thermodynamics. The stability of the phases is a function of the system composition, the monosolvate being the most stable form at THEO:PYR stoichiometries between 1:0.1-1:1 whilst above 1:1 the sesquisolvate becomes the most stable form. In an attempt to unravel the mechanism of solvation, we observed that in the composition ranges where the sesquisolvate becomes the most stable form, sesquisolvate formation occurs via the formation of the monosolvate. Formation of the monosolvate is instantaneous, with $90 \%$ of the monosolvate formed upon seconds of grinding. Formation of the sesquisolvate, however, requires a substantial amount of time (and thus mechanical energy). Attachment energy calculations can provide some understanding as to why the sesquisolvate is formed with much slower kinetics. Upon grinding, crystals are broken and surfaces are created and exposed thus enabling contact with the solvent. This enables the conversion from the reactants to the products. The attachment energy required to expose the key surfaces of THEO Form II was found to be very small and almost four times smaller than the attachment energy required to expose the key surfaces in the monosolvate. This is consistent with a hypothesis that breaking the crystals, thus generating more surfaces that can become in contact with the solvent, is the rate-limiting process in the solvation reaction. Calculations of stronger attachment of cleavage planes in Form IV, relative to Form II, suggest that Form IV should require more mechanical energy to achieve solvate conversion, thus showing slower kinetics. This was found experimentally via manual mixing of reactants as well as via exposure of both forms to PYR vapour.

In summary, we have demonstrated the potential of VALAG as a tool for screening for stoichiometric solvates. We have shown that the solvates obtained by grinding are always the thermodynamic product of the reaction, and the nature and stoichiometry of the thermodynamically stable product depends on the composition. Remarkably, we have correlated the kinetics of mechanochemical reactions with attachment energies of the softest planes in the crystalline reactants. This implies that the process of breaking the crystals and generating new surfaces that come into contact with the solvent is the rate-limiting step in these mechanochemical solvation reactions.

\section{Conflicts of interest}

The authors declare no conflicts of interest.

\section{Acknowledgements}

We thank Dr David Hughes and Professor Tomislav Friscic for valuable scientific discussions. AJCC thanks Professor Mark Spackman for providing access to Crystal Explorer. BG thanks Roche and the University of Manchester for funding. The financial support of AbbVie Laboratories (studentship to GSR) is gratefully acknowledged.

\section{Notes and references}

¥ Although THEO is a commonly used model compound for exploring polymorphism and the formation of multicomponent crystals, so far, with the exception of one monohydrate THEO ${ }^{26}$ and a series of amide solvates ${ }^{43}$ there are no other known solvated crystals for this molecule.

$\S$ THEO was ground in the presence of different solvents during a preliminary screening for new solvates. The results suggested that new PXRD patterns and TGA weight loss, and therefore new solid forms, were obtained only in the experiments with PYR. Understanding the propensity of THEO to form solvates with different solvents is beyond the objectives of this study.

1 U. J. Griesser, B. by Hiliker, 2006, 211-233.

2 H. G. Brittain, J. Pharm. Sci., 2012, 101, 464-484.

3 A. Newman, Org. Process Res. Dev., 2013, 17, 457-471.

$4 \quad$ T. L. Threlfall, Analyst, 1995, 120, 2435-2460.

5 F. P. A. Fabbiani, D. R. Allan, W. I. F. David, S. A. Moggach, S. Parsons and C. R. Pulham, CrystEngComm, 2004, 6, 504511.

6 L. Yu, N. Milton, E. G. Groleau, D. S. Mishra and R. E. Vansickle, J. Pharm. Sci., 1999, 88, 196-198.

$7 \quad$ N. K. Duggirala, M. L. Perry, R. Almarsson and M. J. Zaworotko, Chem. Commun., 2016, 52, 640-655.

8 D. Tan, L. Loots and T. Friščić, Chem. Commun., 2016, 52, 7760-7781.

9 J.-L. Do and T. Friščić, ACS Cent. Sci., 2017, 3, 13-19.

10 E. V Boldyreva, Income 2008, 2011, 17-30.

11 D. Hasa and W. Jones, Adv. Drug Deliv. Rev., 2017, 117, 147-161.

12 G. Coquerel, Chem. Soc. Rev., 2014, 43, 2286-2300.

13 T. Threlfall, Org. Process Res. Dev., 2000, 4, 384-390.

14 V. Stilinović, D. Cinčić, M. Zbačnik and B. Kaitner, Croat. Chem. Acta, 2012, 85, 485-493.

15 S. Karki, T. Friščić, W. Jones and W. D. S. Motherwell, Mol. Pharm., 2007, 4, 347-354. 
P. Wang, G. Li, Y. Chen, S. Chen, S. L. James and W. Yuan, CrystEngComm, 2012, 14, 1994-1997.

I. Sarceviča, I. Grante, S. Belyakov, T. Rekis, K. Bērziņš, A. Actinš and L. Orola, J. Pharm. Sci., 2016, 105, 1489-1495. N. Madusanka, M. D. Eddleston, M. Arhangelskis and W. Jones, Acta Crystallogr. B. Struct. Sci. Cryst. Eng. Mater., 2014, 70, 72-80. M. D. Eddleston, S. Sivachelvam and W. Jones, CrystEngComm, 2013, 15, 175-181. T. Friščić, I. Halasz, F. C. Strobridge, R. E. Dinnebier, R. S. Stein, L. Fábián and C. Curfs, CrystEngComm, 2011, 13, 3125-3129.

G. G. Z. Zhang, D. Law, E. a Schmitt and Y. Qiu, Adv. Drug Deliv. Rev., 2004, 56, 371-390.

F. Tian, H. Qu, A. Zimmermann, T. Munk, A. C. Jørgensen and J. Rantanen, J. Pharm. Pharmacol., 2010, 62, 15341546. S. Karki, T. Friščić and W. Jones, CrystaEngComm, 2009, 11, 470-481. R. J. Davey, P. T. Cardew, D. Mcewan and D. E. Sadler, J. Cryst. Growth, 1986, 79, 648-653.

2015, 17, 5237-5251. K. Fucke, G. J. McIntyre, C. Wilkinson, M. Henry, J. A. K. Howard and J. W. Steed, Cryst. Growth Des., 2012, 12, 1395-1401. G. Kresse and J. Hafner, Phys. Rev. B, 1993, 47, 558-561. G. Kresse and J. Furthmüller, Comput. Mater. Sci., 1996, 6, 15-50.

G. Kresse and J. Furthmüller, Phys. Rev. B, 1996, 54, 11169-11186. J. P. Perdew, K. Burke and M. Ernzerhof, Phys. Rev. Lett., 1996, 77, 3865-3868.

P. E. Blöchl, Phys. Rev. B, 1994, 50, 17953-17979.

G. Kresse and D. Joubert, Phys. Rev. B, 1999, 59, 17581775.

S. Grimme, J. Comput. Chem., 2006, 27, 1787-1799.

M. J. Turner, J. J. McKinnon, S. K. Wolff, D. J. Grimwood, P. R. Spackman, D. Jayatilaka and M. A. Spackman, 2017.

M. J. Turner, S. Grabowsky, D. Jayatilaka and M. A. Spackman, J. Phys. Chem. Lett., 2014, 5, 4249-4255. C. F. Mackenzie, P. R. Spackman, D. Jayatilaka, M. A. Spackman, S. M. A., S. R., S. J., T. R., van de S. J. and W. P. A., IUCrJ, 2017, 4, 575-587.

M. J. Turner, S. P. Thomas, M. W. Shi, D. Jayatilaka and M. A. Spackman, Chem. Commun., 2015, 51, 3691-3928. A. J. Cruz Cabeza, G. M. Day, W. D. S. Motherwell and W. Jones, J. Am. Chem. Soc., 2006, 128, 14466-14467. D. E. Braun, P. G. Karamertzanis and S. L. Price, Chem. Commun., 2011, 47, 5443-5445. A. J. Cruz Cabeza, S. Karki, L. Fábián, T. Friščić, G. M. Day and W. Jones, Chem. Commun., 2010, 46, 2224-2226. A. J. Cruz-Cabeza, G. M. Day and W. Jones, Chem. - A Eur. J., 2008, 14, 8830-8836.

A. M. Belenguer, G. I. Lampronti, A. J. Cruz-Cabeza, C. A.
M. D. Eddleston, M. Arhangelskis, L. Fábián, G. J. Tizzard, S. J. Coles and W. Jones, Cryst. Growth Des., 2016, 16, 51-58. Hunter and J. K. M. Sanders, Chem. Sci., 2016, 7, 66176627. 